\title{
Limit distribution for a semi-Markovian random walk with Weibull distributed interference of chance
}

\author{
Tülay Kesemen ${ }^{1 *}$, Rovshan Aliyevev ${ }^{2,3}$ and Tahir Khaniyev ${ }^{3,4}$
}

\section{"Correspondence:}

tkesemen@gmail.com

${ }^{1}$ Faculty of Science, Department of

Mathematics, Karadeniz Technical

University, Trabzon, 61080, Turkey

Full list of author information is

available at the end of the article

\begin{abstract}
In this paper, a semi-Markovian random walk with a discrete interference of chance $(X(t))$ is considered. In this study, it is assumed that the sequence of random variables $\left\{\zeta_{n}\right\}, n=1,2, \ldots$, which describes the discrete interference of chance, forms an ergodic Markov chain with the Weibull stationary distribution. Under this assumption, the ergodic theorem for the process $X(t)$ is discussed. Then the weak convergence theorem is proved for the ergodic distribution of the process $X(t)$ and the limit form of the ergodic distribution is derived.
\end{abstract}

MSC: 60G50;60K15;60F99

Keywords: semi-Markovian random walk; discrete interference of chance; ergodic distribution; weak convergence; asymptotic expansion; ladder variables

\section{Introduction}

Many interesting problems of stochastic finance, mathematical biology, reliability, queuing, stochastic inventory and mathematical insurance can be expressed by means of random walk processes. Some important studies on this topic exist in literature (see, for example, Aliyev et al. [1-3]; Alsmeyer [4]; Borovkov [5]; Khaniyev et al. [8, 9]; Lotov [10]; Rogozin [11]; Skorohod and Slobodenyuk [12]; Spitzer [13] etc.).

Note that in the studies of Khaniyev et al. [9] and Aliyev et al. [1, 2], the random variables $\zeta_{n}, n=1,2,3, \ldots$, which describe the discrete interference of chance, have exponential, gamma and triangular distribution, respectively, and stationary moments of the ergodic distribution of a semi-Markovian random walk process have been investigated. Moreover, Aliyev et al. [3] and Khaniyev and Atalay [8] investigated a weak convergence theorem for the ergodic distribution of the renewal-reward process when the random variables $\zeta_{n}, n=1,2,3, \ldots$, have gamma and triangular distribution, respectively. In this study, unlike Aliyev et al. [1-3] and Khaniyev et al. [8, 9], we assume that the random variables $\zeta_{n}, n=1,2,3, \ldots$, which describe the discrete interference of chance, are independent and identically distributed random variables with the Weibull distribution, and the weak convergence theorem is proved for the ergodic distribution of a semi-Markovian random walk process, and the limit distribution is derived for the ergodic distribution of the considered process.

This process might be useful in the following situation.

0 2013 Kesemen et al: licensee Springer. This is an Open Access article distributed under the terms of the Creative Commons Attribution License (http://creativecommons.org/licenses/by/2.0), which permits unrestricted use, distribution, and reproduction in any medium, provided the original work is properly cited. 


\section{The model}

Consider a stochastic model, which can be used in the field of insurance. This model can be described as follows.

Suppose that the amount of initial capital of an insurance company is equal to $z \in(0, \infty)$. Assume that the premiums and claims arrive at the insurance company randomly at the times $T_{n}=\sum_{i=1}^{n} \xi_{i}, n \geq 1$, here $\xi_{i}, i \geq 1$, are the random time intervals between two successive claims and premiums. Level of total capital of the company fluctuates in accordance with $\left\{-\eta_{n}\right\}, n \geq 1$. The random variable $\eta_{n}$ expresses difference of claims and premiums, which can take both positive and negative values. The amount of the total capital of the insurance company continues its variation until a random time $\tau_{1}$ which is the time at which the capital level first falls below zero. When the above conditions take place, the amount of the company's capital increases immediately to the level $\zeta_{1}$, which is a random variable having a certain distribution in the interval $(0, \infty)$. Thus, the first period is completed. Then the insurance company keeps working in a way similar to the previous period with a new initial capital $\zeta_{1}$ and so on.

Denote the stochastic process expressed this model mathematically by $X(t)$. Thus, the amount of capital of the insurance company at each time $t$ is represented by the process $X(t)$. The process $X(t)$ is known to be as a semi-Markovian random walk process with a discrete interference of chance.

We now proceed to a mathematical construction of the process $X(t)$.

\section{Mathematical construction of the process $X(t)$}

Let $\left(\xi_{n}, \eta_{n}, \zeta_{n}\right), n \geq 1$ be a sequence of independent and identically distributed vector of random variables, defined on any probability space $(\Omega, \Im, P)$, such that $\xi_{n}$ takes only positive values, $\eta_{n}$ takes positive and negative values as well as positive ones, $\zeta_{n}$ has the Weibull distribution with parameters $(\alpha, \lambda), \alpha>1, \lambda>0$. Suppose that $\xi_{1}, \eta_{1}, \zeta_{1}$ are mutually independent random variables and the distribution functions of them are known, i.e.,

$$
\begin{aligned}
& \Phi(t)=P\left\{\xi_{1} \leq t\right\}, \quad t \geq 0 ; \quad F(x)=P\left\{\eta_{1} \leq x\right\}, \quad x \in(-\infty, \infty) \\
& \pi(z) \equiv P\left\{\zeta_{1} \leq z\right\}=1-\exp \left(-(\lambda z)^{\alpha}\right), \quad z \geq 0, \alpha>1, \lambda>0 .
\end{aligned}
$$

Define the renewal sequence $\left\{T_{n}\right\}$ and the random walk $\left\{S_{n}\right\}$ as follows:

$$
T_{n}=\sum_{i=1}^{n} \xi_{i}, \quad S_{n}=\sum_{i=1}^{n} \eta_{i}, \quad T_{0}=S_{0}=0, \quad n=1,2, \ldots
$$

and define a sequence of integer-valued random variables $\left\{N_{n}\right\}$ as follows:

$$
\begin{aligned}
& N_{0}=0, \quad N_{1} \equiv N(z)=\inf \left\{n \geq 1: z-S_{n}<0\right\}, \quad z \geq 0 ; \\
& N_{n+1}=\inf \left\{k \geq 1: \zeta_{n}-\left(S_{N_{1}+N_{2}+\cdots+N_{n}+k}-S_{N_{1}+N_{2}+\cdots+N_{n}}\right)<0\right\}, \quad n=1,2, \ldots
\end{aligned}
$$

and $\inf \{\emptyset\}=+\infty$ is stipulated.

Let $\tau_{0}=0, \tau_{1} \equiv \tau(z)=T_{N(z)}=\sum_{i=1}^{N(z)} \xi_{i}, z \geq 0 ; \tau_{n}=T_{N_{1}+\cdots+N_{n}}, n \geq 2$, and define $v(t)$ as $v(t)=\max \left\{n \geq 0: T_{n} \leq t\right\}$. 


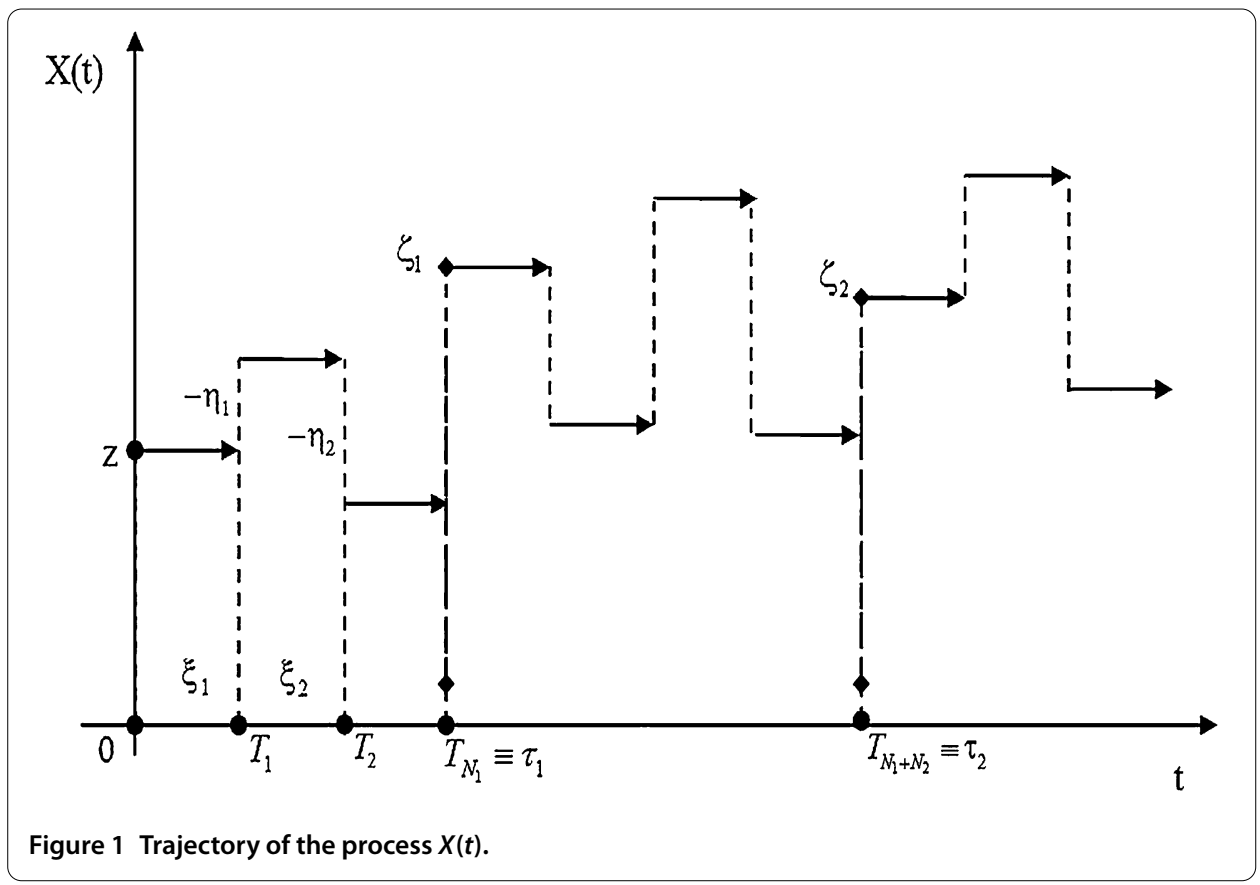

We can now construct the desired stochastic process $X(t)$ as follows:

$$
X(t)=\zeta_{n}-\left(\sum_{i=N_{1}+N_{2}+\cdots+N_{n}+1}^{v(t)} \eta_{i}\right)=\zeta_{n}-\left(S_{v(t)}-S_{N_{0}+N_{1}+\cdots+N_{n}}\right)
$$

if $\tau_{n} \leq t<\tau_{n+1}, n=0,1,2, \ldots ; \zeta_{0} \equiv z \in(0, \infty)$.

Note that the process $X(t)$ describes the amount of the total capital of an insurance company at any time $t \geq 0$.

Figure 1 gives a trajectory of the process $X(t)$.

The main purpose of this study is to prove the weak convergence theorem for the ergodic distribution of the process $X(t)$, as $\lambda \rightarrow 0$. For this aim, we first discuss the ergodicity of the process $X(t)$.

\section{The ergodicity of the process $X(t)$}

State the following proposition on the ergodicity of the process $X(t)$.

Proposition 3.1 (Ergodic theorem [1]) Let the initial sequences of the random variables $\left\{\xi_{n}\right\},\left\{\eta_{n}\right\}$ and $\left\{\zeta_{n}\right\}, n \geq 1$, satisfy the following supplementary conditions:
(1) $E \xi_{1}<\infty$;
(2) $E \eta_{1}>0$;
(3) $E\left(\eta_{1}^{2}\right)<\infty$;

(4) $\eta_{1}$ is non-arithmetic random variable.

Then the process $X(t)$ is ergodic, and for any bounded measurable function $f(x)$; $(f$ : $[0, \infty) \rightarrow R)$, the following relation holds with probability one:

$$
\lim _{t \rightarrow \infty} \frac{1}{t} \int_{0}^{t} f(X(u)) d u=\frac{1}{E(N(\zeta))} \int_{0}^{\infty} f(x) d_{x}(E(A(x, \zeta))) .
$$


Here, $\zeta$ is a random variable with a distribution function $\pi(z)$ and

$$
\begin{aligned}
& E(N(\zeta))=\int_{0}^{\infty} E(N(z)) d \pi(z) ; \quad E(A(x, \zeta))=\int_{0}^{\infty} A(x, z) d \pi(z) \\
& A(x, z)=\sum_{n=0}^{\infty} a_{n}(x, z) ; \quad a_{n}(x, z)=P\left\{z-S_{i}>0, i=\overline{1, n} ; z-S_{n} \leq x\right\}, x>0 ; z>0 .
\end{aligned}
$$

Proof The process $X(t)$ belongs to a wide class of processes which is called 'The class of semi-Markov processes with a discrete interference of chance' in literature. General ergodic theorem of type 'Smith's key renewal theorem' exists in literature for this class (see, Gihman and Skorohod [7], p.243). It is not difficult to show that the assumptions of the general ergodic theorem are satisfied under the conditions of Proposition 3.1. Therefore, the ergodicity of the process $X(t)$ is derived by using this general ergodic theorem.

Corollary 3.1 The ergodic distribution function $\left(Q_{X}(x)\right)$ of the process $X(t)$ can be written as follows:

$$
Q_{X}(x) \equiv \lim _{t \rightarrow \infty} P\{X(t) \leq x\}=\frac{E(A(x, \zeta))}{E(N(\zeta))}, \quad x>0
$$

Proof Substituting the indicator function instead of the $f(x)$ in Eq. (1), we can obtain Eq. (2).

Now we define the characteristic function of the ergodic distribution of the process $X(t)$ as follows: $\varphi_{X}(u) \equiv \lim _{t \rightarrow \infty} E\{\exp (i u X(t))\}, u \in R$.

Corollary 3.2 The characteristic function $\left(\varphi_{X}(u)\right)$ of the ergodic distribution of the process $X(t)$ can be represented as follows:

$$
\varphi_{X}(u) \equiv \lim _{t \rightarrow \infty} E(\exp (i u X(t)))=\frac{1}{E(N(\zeta))} \int_{0}^{\infty} e^{i u x} d_{x} E(A(x, \zeta)), \quad u \in R
$$

Using the basic identity for the random walks (see, Feller [6], p.514), from Eq. (3) we obtain the following lemma.

Lemma 3.1 Let the conditions of Proposition 3.1 be satisfied. Then, for each $u \in R /\{0\}$, the characteristic function $\varphi_{X}(u)$ of the ergodic distribution of the process $X(t)$ can be expressed by means of the characteristics of the pair $\left(N(z), S_{N(z)}\right)$ and the random variable $\eta_{1}$ as follows:

$$
\varphi_{X}(u)=\frac{\alpha \lambda^{\alpha}}{E N(\zeta)} \int_{0}^{\infty} z^{\alpha-1} e^{-(\lambda z)^{\alpha}} e^{i u z} \frac{\varphi_{S_{N(z)}}(-u)-1}{\varphi_{\eta}(-u)-1} d z
$$

where $E N(\zeta) \equiv \alpha \lambda^{\alpha} \int_{0}^{\infty} z^{\alpha-1} e^{-(\lambda z)^{\alpha}} E N(z) d z ; \varphi_{S_{N(z)}}(-u)=E \exp \left(-i u S_{N(z)}\right) ; \varphi_{\eta}(-u)=E \times$ $\exp \left(-i u \eta_{1}\right)$.

\section{Weak convergence theorem for the ergodic distribution of the process $X(t)$}

In this section, we use the ladder variables of the random walk $S_{n}=\sum_{i=1}^{n} \eta_{i}, n \geq 1$, with the initial state $S_{0}=0$. Let $v_{1}^{+}=\min \left\{n \geq 1: S_{n}>0\right\}, \chi_{1}^{+}=S_{v_{1}^{+}}=\sum_{i=1}^{v_{1}^{+}} \eta_{i}$. 
Note that the random variables $\nu_{1}^{+}$and $\chi_{1}^{+}$are called the first ascending ladder epoch and ladder height of the random walk $\left\{S_{n}\right\}, n \geq 0$, respectively (see, Feller [6], p.391). Define $\left\{\chi_{n}^{+}\right\}, n \geq 2$, as the sequence of independent random variables having the same distribution with $\chi_{1}^{+}$. Denote by $H(x)$ a renewal process generated by the sequence of random variables $\left\{\chi_{n}^{+}\right\}, n \geq 1$, i.e.,

$$
H(x)=\min \left\{n \geq 1: \sum_{i=1}^{n} \chi_{i}^{+}>x\right\}, \quad x \geq 0 .
$$

For shortness, put $E(H(x)) \equiv U_{+}(x)$. It is known that

$$
U_{+}(x)=1+\sum_{n=1}^{\infty} F_{+}^{* n}(x), \quad x \geq 0,
$$

where $F_{+}^{* n}(x)$ is $n$-fold convolution of the distribution function $F_{+}(x) \equiv P\left\{\chi_{1}^{+} \leq x\right\}$.

Our aim is to prove the weak convergence theorem for the ergodic distribution as $E(\zeta) \rightarrow \infty$. For the Weibull distribution, it is known that $E(\zeta)=\frac{\Gamma(1+(1 / \alpha))}{\lambda}$. In this study, $\alpha$ will be fixed. Therefore while $E(\zeta) \rightarrow \infty$, the parameter $\lambda$ should converge to zero. Hence, we need to give the following lemma first.

Lemma 4.1 Let $g(x)\left(g: R^{+} \rightarrow R\right)$ be a measurable and bounded function, and let $\lim _{x \rightarrow \infty} g(x)=0$. Then, for each $\alpha>1$, the following asymptotic relationship holds:

$$
\lim _{\lambda \rightarrow 0} \int_{0}^{\infty} e^{-t} g\left(\frac{t^{1 / \alpha}}{\lambda}\right) d t=0
$$

Proof Under the conditions of Lemma 4.1, for any $\varepsilon>0$, there exists $m(\varepsilon)>0$ such that for any $x \geq m(\varepsilon)$, the inequality $|g(x)|<\varepsilon$ holds. Choose $b>0$, such that $\int_{0}^{b} e^{-t} d t<\varepsilon$. The function $g(x)$ is bounded. Therefore, for any $\lambda<\frac{b^{1 / \alpha}}{m(\varepsilon)}$, we have

$$
\begin{aligned}
\left|\int_{0}^{\infty} e^{-t} g\left(\frac{t^{1 / \alpha}}{\lambda}\right) d t\right| & \leq \int_{0}^{b} e^{-t}\left|g\left(\frac{t^{1 / \alpha}}{\lambda}\right)\right| d t+\int_{b}^{\infty} e^{-t}\left|g\left(\frac{t^{1 / \alpha}}{\lambda}\right)\right| d t \\
& \leq M \int_{0}^{b} e^{-t} d t+\varepsilon \int_{b}^{\infty} e^{-t} d t \leq \varepsilon M+\varepsilon \int_{0}^{\infty} e^{-t} d t=\varepsilon(M+1),
\end{aligned}
$$

where $M=\max _{x \geq 0}|g(x)|$.

Since $M$ is finite and $\varepsilon>0$ is an arbitrary positive number, the proof of Lemma 4.1 is completed.

We can give the following lemma, which has a similar proof to that of Lemma 4.1.

Lemma 4.2 Let $g(x)$ be defined as in Lemma 4.1 and the function $R_{n}(x)$ be defined as $R_{n}(x) \equiv x^{n} g(x), n=-1,0,1,2, \ldots$ Then, for each $\alpha>1$, the following asymptotic relationship is true, as $\lambda \rightarrow 0$ :

$$
\int_{0}^{\infty} e^{-t} R_{n}\left(\frac{t^{1 / \alpha}}{\lambda}\right) d t=o\left(\frac{1}{\lambda^{n}}\right)
$$


For the investigation of the asymptotic behavior of the ergodic distribution of the process $X(t)$ as $\lambda \rightarrow 0$, we define the auxiliary process $W_{\lambda}(t)$ as $W_{\lambda}(t) \equiv \lambda X(t)$ and investigate the asymptotic behavior of its ergodic distribution function. It is easily seen that the process $W_{\lambda}(t)$ is a linear transform of $X(t)$. Therefore, from Proposition 3.1, it is immediately follows that the process $W_{\lambda}(t)$ is also ergodic under the conditions of Proposition 3.1. Let us denote the characteristic function of the ergodic distribution of $W_{\lambda}(t)$ by $\varphi_{w_{\lambda}}(u)$ and formulate the following statement.

Theorem 4.1 Let the conditions of Proposition 3.1 be satisfied. Then, for each $x>0$ and for all $\alpha>1$, the family of ergodic distribution functions $Q_{W_{\lambda}}(x) \equiv \lim _{t \rightarrow \infty} P\left\{W_{\lambda}(t) \leq x\right\}$ of the process $W_{\lambda}(t)$ weakly converges to a limit distribution function $G(x)$ as follows, when $\lambda \rightarrow 0$ :

$$
Q_{W_{\lambda}}(x) \rightarrow G(x) \equiv \frac{1}{\Gamma(1+1 / \alpha)} \int_{0}^{x} \exp \left(-u^{\alpha}\right) d u
$$

where $\Gamma(\alpha)=\int_{0}^{\infty} t^{\alpha-1} e^{-t} d t$ is Euler's gamma function.

Note that $G(x)$ is the limit distribution of a 'residual waiting time' (see, Feller [6], p.368).

Proof From Eq. (4) it is easily seen that the characteristic function of the process $W_{\lambda}(t)$ can be written as follows:

$$
\varphi_{W_{\lambda}}(u)=\frac{1}{I_{1}(\alpha, \lambda)}\left[I_{2}(\alpha, \lambda)-I_{3}(\alpha, \lambda)\right]
$$

where $I_{1}(\alpha, \lambda)=E N(\zeta)\left[1-\varphi_{\eta}(-\lambda u)\right], I_{2}(\alpha, \lambda)=\alpha \lambda^{\alpha} \int_{0}^{\infty} x^{\alpha-1} e^{-(\lambda x)^{\alpha}}\left[e^{i \lambda u x}-1\right] d x$,

$$
I_{3}(\alpha, \lambda)=\alpha \lambda^{\alpha} \int_{0}^{\infty} x^{\alpha-1} e^{-(\lambda x)^{\alpha}} E\left[\exp \left(-i u \lambda \bar{S}_{N(x)}\right)-1\right] d x, \quad \bar{S}_{N(x)} \equiv S_{N(x)}-x, x>0
$$

From the sharper form of the renewal theorem (see, Feller [6], p.366) we get, when $\lambda \rightarrow 0$ :

$$
\operatorname{EN}(\zeta)=\frac{1}{m_{1}} \frac{\Gamma(1+(1 / \alpha))}{\lambda}+\frac{m_{2}}{2 m_{1}^{2}}+J(\alpha, \lambda)
$$

where $J(\alpha, \lambda)=\alpha \lambda^{\alpha} \int_{0}^{\infty} x^{\alpha-1} e^{-(\lambda x)^{\alpha}} g(x) d x$.

From Lemma 4.1, as $\lambda \rightarrow 0$, for all $\alpha>1$, we have

$$
J(\alpha, \lambda)=o(1)
$$

Substituting $J(\alpha, \lambda)$ in Eq. (6), we get, as $\lambda \rightarrow 0$ :

$$
E N(\zeta)=\frac{1}{m_{1}} \frac{\Gamma(1+(1 / \alpha))}{\lambda}+\frac{m_{2}}{2 m_{1}^{2}}+o(1)
$$

On the other hand, as $\lambda \rightarrow 0$,

$$
1-\varphi_{\eta}(-\lambda u)=i \lambda u m_{1}[1+o(1)]
$$


Thus,

$$
I_{1}(\alpha, \lambda)=i u \Gamma(1+1 / \alpha)[1+o(1)] .
$$

It can be seen that

$$
I_{2}(\alpha, \lambda)=\varphi_{\alpha, 1}(u)-1,
$$

where $\varphi_{\alpha, 1}(u)$ is the characteristic function of the Weibull distribution with parameter $(\alpha, 1)$.

Now we can investigate the asymptotic behavior of $I_{3}(\alpha, \lambda)$ as $\lambda \rightarrow 0$.

The conditions $E \eta_{1}>0$ and $E \eta_{1}^{2}<\infty$ ensure that $\mu_{2} \equiv E\left(\chi_{1}^{+}\right)^{2}$ is finite.

By a sharper form of the renewal theorem (see, Feller [6], p.366), as $x \rightarrow \infty$,

$$
E\left(\bar{S}_{N(x)}\right)=\frac{\mu_{2}}{2 \mu_{1}^{2}}+o(1)
$$

where $\bar{S}_{N(x)} \equiv S_{N(x)}-x, \mu_{k}=E\left(\chi_{1}^{+}\right)^{k}, k=1,2$.

By using Eq. (10) and Lemma 4.1, we get, as $\lambda \rightarrow 0$ :

$$
\alpha \lambda^{\alpha} \int_{0}^{\infty} x^{\alpha-1} e^{-(\lambda x)^{\alpha}} E\left(\bar{S}_{N(x)}\right) d x=\frac{\mu_{2}}{2 \mu_{1}^{2}}+o(1) .
$$

On the other hand,

$$
\left|E\left(\exp \left(-i u \lambda \bar{S}_{N(x)}\right)\right)-1\right| \leq|\lambda u| E\left(\bar{S}_{N(x)}\right) .
$$

By using Eq. (11) and Eq. (12), we get, as $\lambda \rightarrow 0$ :

$$
\left|I_{3}(\alpha, \lambda)\right|=\alpha \lambda^{\alpha}\left|\int_{0}^{\infty} x^{\alpha-1} e^{-(\lambda x)^{\alpha}} E\left[\exp \left(-i u \lambda \bar{S}_{N(x)}\right)-1\right] d x\right| \leq \lambda|u|\left[\frac{\mu_{2}}{2 \mu_{1}^{2}}+o(1)\right] .
$$

Thus, we obtain, as $\lambda \rightarrow 0$ :

$$
I_{3}(\alpha, \lambda)=O(\lambda)
$$

From Eq. (8), Eq. (9) and Eq. (13) for all $\alpha>1$, we get, as $\lambda \rightarrow 0$ :

$$
\varphi_{W_{\lambda}}(u)=\frac{\varphi_{\alpha, 1}(u)-1}{i u \Gamma(1+(1 / \alpha))}[1+o(1)] .
$$

It means that the family of characteristic functions $\left\{\varphi_{W_{\lambda}}(u)\right\}$ converges to a limit characteristic function $\frac{\varphi_{\alpha, 1}(u)-1}{i u \Gamma(1+1 / \alpha)}$. Here $\varphi_{\alpha, 1}(u)$ is the characteristic function of the Weibull distribution with parameter $(\alpha, 1)$.

Therefore, by the continuity theorem for characteristic functions (see, Feller [6], p.508), the ergodic distribution function of the process $W_{\lambda}(t)$ weakly converges to the limit distribution function $G(x) \equiv \frac{1}{\Gamma(1+(1 / \alpha))} \int_{0}^{x} \exp \left(-u^{\alpha}\right) d u$ as $\lambda \rightarrow 0$ uniformly for $x>0$, i.e.,

$$
\lim _{\lambda \rightarrow 0} Q_{W_{\lambda}}(x)=G(x) \equiv \frac{1}{\Gamma(1+1 / \alpha)} \int_{0}^{x} \exp \left(-u^{\alpha}\right) d u .
$$

This completes the proof of Theorem 4.1. 
Note that $G(x)$ is the limit distribution for the 'residual waiting time' generating by the sequence $\left\{\zeta_{n}\right\}$ (see, Feller [6], p.386).

\section{Conclusion}

In this paper, a semi-Markovian random walk with a discrete interference of chance $(X(t))$ is considered and the ergodic theorem for this process is discussed under some conditions. Finally, the weak convergence theorem is proved for the ergodic distribution of the process $W_{\lambda}(t) \equiv \lambda X(t)$, and the limit form of the ergodic distribution $G(x)$ is established. Note that $G(x)$ is a limit distribution of a residual waiting time of a renewal process generated by the random variables having the Weibull distribution with parameters $(\alpha, 1)$. In the terms of insurance, here we derived the explicit form of the limit distribution of the amount of the capital of an insurance company which is working for a long time as $E(\zeta) \rightarrow \infty$.

Competing interests

The authors declare that they have no competing interests.

Authors' contributions

The present study was proposed by TK. All authors read and approved the final manuscript.

\section{Author details}

${ }^{1}$ Faculty of Science, Department of Mathematics, Karadeniz Technical University, Trabzon, 61080, Turkey. ${ }^{2}$ Department of Probability Theory and Mathematical Statistics, Baku State University, Z. Khalilov Str. 23, AZ 1148, Baku, Azerbaijan.

${ }^{3}$ Institute of Cybernetics of Azerbaijan National Academy of Sciences, F. Agayev Str. 9, AZ 1141, Baku, Azerbaijan.

${ }^{4}$ Department of Industrial Engineering, TOBB University of Economics and Technology, Sögütözü, Ankara 06560, Turkey.

\section{Acknowledgements}

Dedicated to Professor Hari M Srivastava.

Received: 23 November 2012 Accepted: 12 March 2013 Published: 29 March 2013

\section{References}

1. Aliyev, RT, Khaniyev, TA, Kesemen, T: Asymptotic expansions for the moments of a semi-Markovian random walk with gamma distributed interference of chance. Commun. Stat., Theory Methods 39, 130-143 (2010)

2. Aliyev, R, Kucuk, Z, Khaniyev, T: Three-term asymptotic expansions for the moments of the random walk with triangular distributed interference of chance. Appl. Math. Model. 34(11), 3599-3607 (2010)

3. Aliyev, RT, Khaniyev, TA, Bekar, NO: Weak convergence theorem for the ergodic distribution of the renewal-reward with gamma distributed interference of chance. Theory Stoch. Process. 15(31)(2), 42-53 (2009)

4. Alsmeyer, G: Some relations between harmonic renewal measure and certain first passage times. Stat. Probab. Lett. 12(1), 19-27 (1991)

5. Borovkov, AA: Stochastic Process in Queuing Theory. Springer, New York (1976)

6. Feller, W: Introduction to Probability Theory and Its Applications II. Wiley, New York (1971)

7. Gihman, II, Skorohod, AV: Theory of Stochastic Processes II. Springer, Berlin (1975)

8. Khaniyev, TA, Atalay, KD: On the weak convergence theorem for the ergodic distribution for an inventory model of type (S, S). Hacet. J. Math. Stat. 39(4), 599-611 (2010)

9. Khaniyev, TA, Kesemen, T, Aliyev, RT, Kokangul, A: Asymptotic expansions for the moments of a semi-Markovian random walk with exponential distributed interference of chance. Stat. Probab. Lett. 78(6), 785-793 (2008)

10. Lotov, VI: On some boundary crossing problems for Gaussian random walks. Ann. Probab. 24(4), 2154-2171 (1996)

11. Rogozin, BA: On the distribution of the first jump. Theory Probab. Appl. 9(3), 498-545 (1964)

12. Skorohod, AV, Slobodenyuk, NP: Limit Theorems for the Random Walks. Naukova Dumka, Kiev (1970)

13. Spitzer, F: Principles of Random Walks. Van Nostrand, Princeton (1964)

doi:10.1186/1029-242X-2013-134

Cite this article as: Kesemen et al.: Limit distribution for a semi-Markovian random walk with Weibull distributed interference of chance. Journal of Inequalities and Applications 2013 2013:134. 